\title{
The p38 MAPK inhibitor SB203580 differentially modulates LPS-induced interleukin 6 expression in macrophages
}

\author{
QINGHAI SHI, LIPING CHENG, ZHENGXIANG LIU, KEYAN HU, JIHUA RAN, DI GE, JIANFENG FU
}

Clinical Laboratory Diagnostic Center, Urumqi General Hospital of PLA, China

\begin{abstract}
The p38 mitogen-activated protein kinase (MAPK) plays a key role in lipopolysaccharide (LPS)-induced signal transduction pathways that lead to inflammatory cytokine synthesis in macrophages; however, whether the inhibition of 338 MAPK regulates LPS-induced inflammatory cytokine expression in different types of macrophages remains the subject of debate. Herein, we assessed whether the inhibition of p38 MAPK by SB203580 regulates LPS-induced expression of the inflammatory cytokines tumor necrosis factor $\alpha(T N F-\alpha)$ and interleukin $6(I L-6)$ in RAW264.7 and resident peritoneal macrophages. Lipopolysaccharide stimulation of RAW264.7 macrophages or mouse resident peritoneal macrophages significantly increased TNF- $\alpha$ and IL-6 production. The addition of SB203580 to cultures dramatically blocked LPS-induced TNF- $\alpha$ production in RAW264.7 and mouse resident peritoneal macrophages, and dramatically blocked LPS-induced IL-6 production in RAW264.7 macrophages, but not in mouse resident peritoneal macrophages. Additionally, high concentrations of $S B 203580$ resulted in increased IL-6 production. However, LPS-stimulation significantly up-regulated the $m R N A$ transcript levels of $T N F-\alpha$ and IL-6 in RAW264.7 and mouse resident peritoneal macrophages, whereas pretreatment with SB203580 dramatically down-regulated LPS-induced mRNA transcript levels of TNF- $\alpha$ and IL-6 in these cells. Our data show that SB203580 differentially modulates LPS-induced production of the inflammatory cytokine IL-6 in two different sources of macrophages, and that this course of regulation occurs at the IL-6 mRNA post-transcriptional stage.
\end{abstract}

Key words: p38, interleukin 6, cytokine, lipopolysaccharide, macrophage.

(Cent Eur J Immunol 2015; 40 (3): 276-282)

\section{Introduction}

Macrophages play an important role in host defense against microbial and viral infections. Activation of macrophages by lipopolysaccharide (LPS, also known as endotoxin), the major constituent of the outer membrane of Gram-negative bacteria, and the consequent expression of many inflammatory genes induced by Toll-like receptor (TLR)-mediated intracellular signaling cascades, which include non-receptor protein tyrosine kinases and serine/ threonine protein kinases, such as mitogen-activated protein kinases (MAPKs; e.g., extracellular signal regulated kinase [ERK], c-Jun N-terminal kinase [JNK], and p38). The p38 MAPK is an important member of the MAPK family that plays key roles in LPS-induced signal transduction pathways and leads to the synthesis of inflammatory cytokines, such as tumor necrosis factor $\alpha$ (TNF- $\alpha)$ and various interleukins (ILs). Therefore, it might represent a promising therapeutic target in a broad range of inflammatory diseases.

SB203580 is a pyridinyl imidazole compound that is a selective ATP-competitive inhibitor of p38 MAPK. Several previous studies showed that SB203580 could inhibit the production of TNF- $\alpha$ and IL-6 in LPS-stimulated macrophages. However, Kim et al. reported that SB203580 could increase LPS-induced TNF- $\alpha$ and interleukin (IL)12 mRNA expression, but reduced the mRNA expression levels of IL-1 $\beta$ and IL-6 in J774A.1 macrophages. Li et al. reported that LPS induced the production of IL-1 $\beta$, TNF- $\alpha$, and IL-6 in mouse bone marrow-derived macrophages, and SB203580 failed to inhibit LPS-induced cytokine production. Finally, Page et al. reported that SB203580 inhibited TNF- $\alpha$ production, but increased IL- 6 and IL-8 production by LPS-stimulated primary human macrophages. In summary, these previous studies have yielded inconsistent data regarding whether SB203580 can regulate expression

Correspondence: Prof. Jianfeng Fu, Clinical Laboratory Diagnostic Center, Urumqi General Hospital of PLA, China, e-mail: dxpjf@163.com 
of the inflammatory cytokines TNF- $\alpha$ and IL-6 induced by LPS in various types of macrophages. Herein, we also demonstrate that the p38 MAPK inhibitor SB203580 differentially modulates LPS-induced production of the inflammatory cytokine IL-6 in RAW 264.7 macrophages and resident peritoneal macrophages, and this regulation occurs at the IL-6 mRNA post-transcriptional stage.

\section{Material and methods}

\section{Reagents}

SB203580, lipopolysaccharide (LPS) from Escherichia coli (serotype O111:B4), 3-(4,5-dimethylthiazol-2-yl)-2,5-diphenyltetrazolium (MTT) and DMSO was obtained from Sigma Chemicals (St. Louis, MO, USA). Antibody against phosphor-p38 (p-p38) (Cat. No.: \#4511) was obtained from Cell Signaling Technology (Danvers, MA, USA) and antibody against $\beta$-actin (Cat. No.: sc47778) from Santa Cruz Biotechnologies (Santa Cruz, CA, USA). All the other chemicals were of reagent grade or the highest quality available and were from Sigma.

\section{Cell culture}

RAW 264.7 macrophages. Murine macrophage RAW 264.7 cells were purchased from the American Type Culture Collection (Manassas, VA, USA) and were seeded into 96-well plates at a density of $5 \times 10^{5}$ cells per well in Dulbecco modified Eagle medium (DMEM) supplemented with $10 \%$ low-endotoxin fetal calf serum (HyClone, Logan, UT), $2 \mu \mathrm{m}$ of glutamine, $100 \mathrm{U}$ of penicillin/ml, and $100 \mu \mathrm{g}$ of streptomycin $/ \mathrm{ml}$ in a $37^{\circ} \mathrm{C}$ humid atmosphere containing $5 \% \mathrm{CO}_{2}$.

Primary mice macrophages. Resident peritoneal macrophages from KM male mice (Animal Center of the Xinjiang Medical University, 10-12-week-old) were obtained by peritoneal lavage using $5 \mathrm{ml}$ of cold PBS, as described elsewhere. The cells were seeded at a density of $5 \times 10^{5}$ cells per well in 96-well plates in DMEM supplemented with $10 \%$ low-endotoxin fetal calf serum, $2 \mu \mathrm{m}$ of glutamine, $100 \mathrm{U}$ of penicillin/ml, and $100 \mu \mathrm{g}$ of streptomycin/ $\mathrm{ml}$ and allowed to adhere for $20 \mathrm{~h}$ in a $37^{\circ} \mathrm{C}$ humid atmosphere containing $5 \% \mathrm{CO}_{2}$. Wells were washed extensively with PBS to remove non-adherent cells. Adherent macrophages were used for further experimentation. All procedures involving animals were undertaken in accordance with the Provisions and General Recommendation of the Chinese Experimental Animals Administration Legislation and were approved by the Science and Technology Department of Xinjiang Province.

\section{MTT assay}

Cell viability was evaluated using an MTT assay. The cells were seeded at a density of $1 \times 10^{4}$ cells per well in a 96-well plates and maintained at $37^{\circ} \mathrm{C}$ for $24 \mathrm{~h}$. The cells were exposed to various concentrations of SB203580 (0.5, $1,2,4,8$, and $16 \mu \mathrm{M})$ for $1 \mathrm{~h}$ and then stimulated with LPS $(50 \mathrm{ng} / \mathrm{ml})$. After $24 \mathrm{~h}$ of incubation, the MTT reagent $(0.5 \mathrm{mg} / \mathrm{ml}$ in DPBS $)$ was added to the maintenance cell medium and incubated at $37^{\circ} \mathrm{C}$ for an additional $4 \mathrm{~h}$. The reaction was terminated with $150 \mu$ of DMSO per well, the cells were lysed for $15 \mathrm{~min}$, and the plates were gently agitated for $5 \mathrm{~min}$. The absorbance values were determined using an ELISA reader (Model 550; Bio-Rad, Hercules, CA, USA) at $490 \mathrm{~nm}$.

\section{Enzyme-linked immunosorbent assay (ELISA)}

The cells were seeded in 96-well plates and treated for $1 \mathrm{~h}$ with SB203580 and stimulated for another $12 \mathrm{~h}$ with LPS $(50 \mathrm{ng} / \mathrm{ml})$. Then, culture supernatants were collected and examined for concentrations of TNF- $\alpha$ and IL- 6 by ELISA kits (Biosource International), following the manufacturer's instructions. The absorbance was measured at a wavelength of $450 \mathrm{~nm}$ using a Model 550 microplate reader.

\section{Quantitative real-time reverse transcriptase PCR}

The cells were seeded in 6-well plates and treated for $1 \mathrm{~h}$ with SB203580 and stimulated for another $4 \mathrm{~h}$ with LPS (50 ng/ml). Total RNA was extracted using an Easy Total RNA Extraction Kit (Tiangen, China) according to the manufacturer's instructions. Total RNA $(1 \mu \mathrm{g})$ was converted to cDNA using the ReverTra ACE- $\alpha$-RNAeasy kit (Toyobo Co Ltd., Japan). Quantitative real-time reverse transcriptase PCR (RT-PCR) analysis for TNF- $\alpha$, IL- 6 and $\beta$-actin was performed using a Bio-Rad CFX96 real-time system. Quantitative PCR was conducted in 0.2-ml PCR tubes with the appropriate forward and reverse primers and the SYBR green working solution (Toyobo Co Ltd., Japan), using a custom PCR master mix under the following conditions: $95^{\circ} \mathrm{C}$ for $60 \mathrm{~s}$, followed by 40 cycles of $95^{\circ} \mathrm{C}$ for $15 \mathrm{~s}, 59^{\circ} \mathrm{C}$ for $15 \mathrm{~s}$ and $72^{\circ} \mathrm{C}$ for $45 \mathrm{~s}$. The following primers were used: TNF- $\alpha$, forward 5'-CAGGTTCTGTCCCTTTCACTCACT-3' and reverse 5'-GTTCAGTAGACAGAAGAGCGTGGT-3'; IL-6, forward 5'-ACCACGGCCTTCCCTACTT-3' and reverse 5'-CATTTCCACGATTTCCCAGA-3'; $\beta$-actin, forward 5'-CTGTCCCTGTATGCCTCTG-3' and reverse 5'-ATGTCACGCACGATTTCC-3'. All quantitations were normalized to $\beta$-actin. Relative quantitation was performed using the $\Delta \Delta \mathrm{C}_{\mathrm{t}}$ method according to the manufacturer's instructions.

\section{Western blotting}

The cells were seeded in 6-well plates and pretreated with SB203580 for $1 \mathrm{~h}$ and stimulated with LPS (50 ng/ $\mathrm{ml})$ for $30 \mathrm{~min}$. Then, the cells were washed with ice-cold DPBS and suspended in $250 \mu$ of lysis buffer $(10 \mathrm{mM}$ 
Tris- $\mathrm{HCl}$ [pH 7.6], $140 \mathrm{mM} \mathrm{NaCl}, 1 \mathrm{mM}$ phenylmethylsulphonyl fluoride, $1 \%$ Nonidet P-40, $0.5 \%$ deoxycholate, $2 \% \beta$-mercaptoethanol, $10 \mu \mathrm{g} / \mathrm{ml}$ pepstatin A and $10 \mu \mathrm{g} /$ $\mathrm{ml}$ aprotinin) and kept at $4^{\circ} \mathrm{C}$ for $15 \mathrm{~min}$. The homogenate was centrifuged at $13,000 \mathrm{~g}$ for $10 \mathrm{~min}$ at $4^{\circ} \mathrm{C}$. The supernatant was collected, and the protein content of the lysates was estimated using an enhanced BCA protein assay kit (Beyotime, Haimen, China). Sample proteins $(20 \mu \mathrm{g})$ were resolved by sodium dodecyl sulfate-polyacrylamide gel electrophoresis (SDS-PAGE) and transferred onto a hybrid polyvinylidene difluoride (PVDF) membrane. Non-specific protein binding was blocked by saturating the PVDF membranes with $5 \%$ bovine serum albumin (BSA) at room temperature for $1 \mathrm{~h}$ and then incubating them with primary antibodies against p-p38 $(1: 1000)$ or $\beta$-actin $(1: 200)$ overnight at $4^{\circ} \mathrm{C}$. After three washes with a Tris-buffered saline solution containing $0.1 \%$ Tween 20 (TBST), the membranes were incubated at room temperature for $1 \mathrm{~h}$ with HRP-conjugated goat anti-rabbit IgG antibody (diluted $1: 1,000)$ or HRP-conjugated goat anti-mouse IgG antibody (diluted $1: 1,000$ ), and then the immunoreactivity was visualized using a chemiluminescent peroxidase substrate kit according to the manufacturer's instructions (Pierce, USA).

\section{Statistical analysis}

All experiments were repeated 2-3 times with similar trends, however, data from a representative experiment are depicted in the results. The data are expressed as the mean \pm standard error. One-way ANOVA followed by the least-significant difference (LSD) multiple group comparison was used to analyze group differences in the data. A value of $p<0.05$ was used to indicate significant differences.

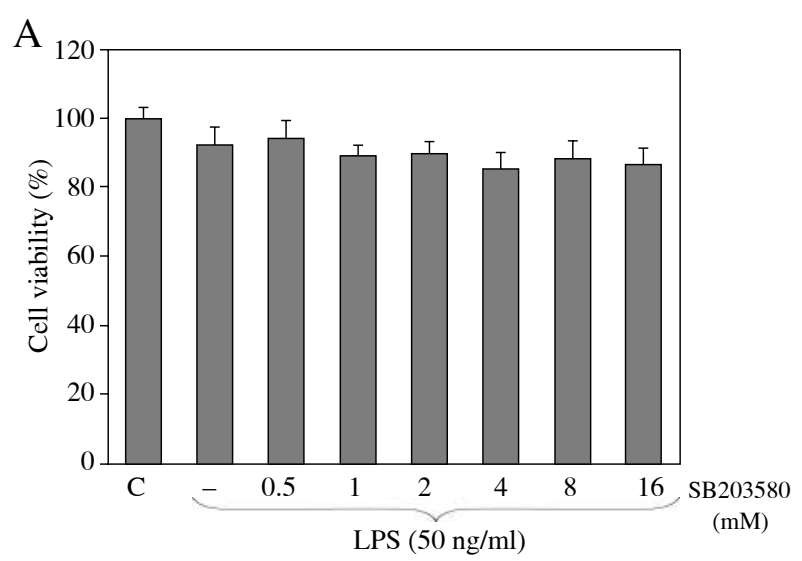

\section{Results}

\section{Kinase inhibitory properties of SB203580 in macrophages}

We first measured the cytotoxicity of SB203580 in RAW264.7 macrophages and mouse resident peritoneal macrophages using the MTT assay. Cell viability was not affected at $24 \mathrm{~h}$ treatment with SB203580 at concentrations up to $16 \mu \mathrm{M}$ in both RAW264.7 and mouse resident peritoneal macrophages (Fig. 1A, B). In subsequent experiments designed to assess kinase inhibitory properties, the concentrations SB203580 used herein caused the partial inhibition of p38 MAPK in RAW264.7 and mouse resident peritoneal macrophages (Fig. 2A, B).

\section{Inhibition of p38 MAPK differentially modulates LPS-induced inflammatory cytokine production in macrophages}

The activity of p38 MAPK can modulate inflammatory cytokine production. To assess the differential modulation of various cytokines by $\mathrm{p} 38$ MAPK, we measured TNF- $\alpha$ and IL-6 production. Stimulation of RAW264.7 macrophages and mouse resident peritoneal macrophages with LPS significantly increased the production of TNF- $\alpha$ and IL-6 (Fig. 3). Pretreatment with SB203580 dramatically blocked LPS-induced TNF- $\alpha$ production in RAW264.7 and mouse resident peritoneal macrophages (Fig. 3A, C). By contrast, pretreatment with SB203580 dramatically blocked LPS-induced IL-6 production in RAW264.7 macrophages, but failed to do the same in mouse resident peritoneal macrophages (Fig. 3B, D). Additionally, high SB203580 concentrations resulted in increased IL-6 production in mouse resident peritoneal macrophages (Fig. 3D).

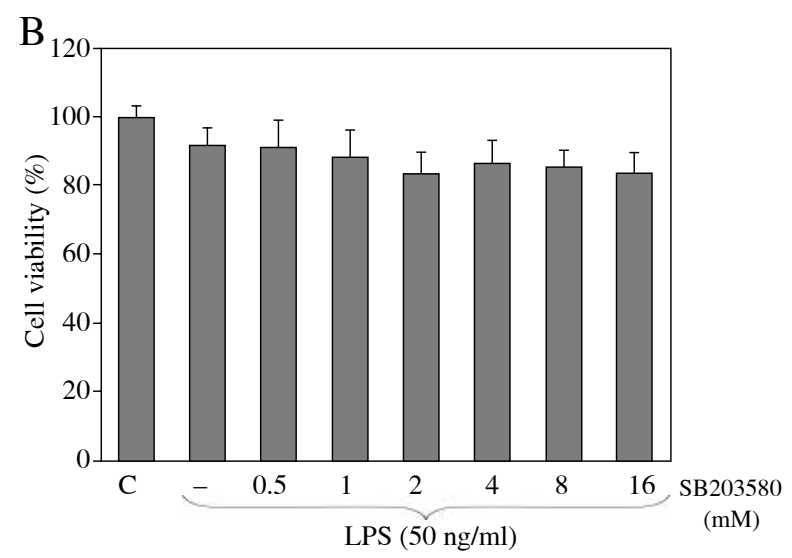

Fig. 1. The viability of (A) RAW 264.7 macrophages and (B) mouse resident peritoneal macrophages cultured in the presence or absence of SB203580 $(0.5,1,2,4,8$, or $16 \mu \mathrm{M})$ and stimulated with LPS $(50 \mathrm{ng} / \mathrm{ml})$ for $24 \mathrm{~h}$. SB203580 did not show cytotoxicity at concentrations of up to $16 \mu \mathrm{M}$ 

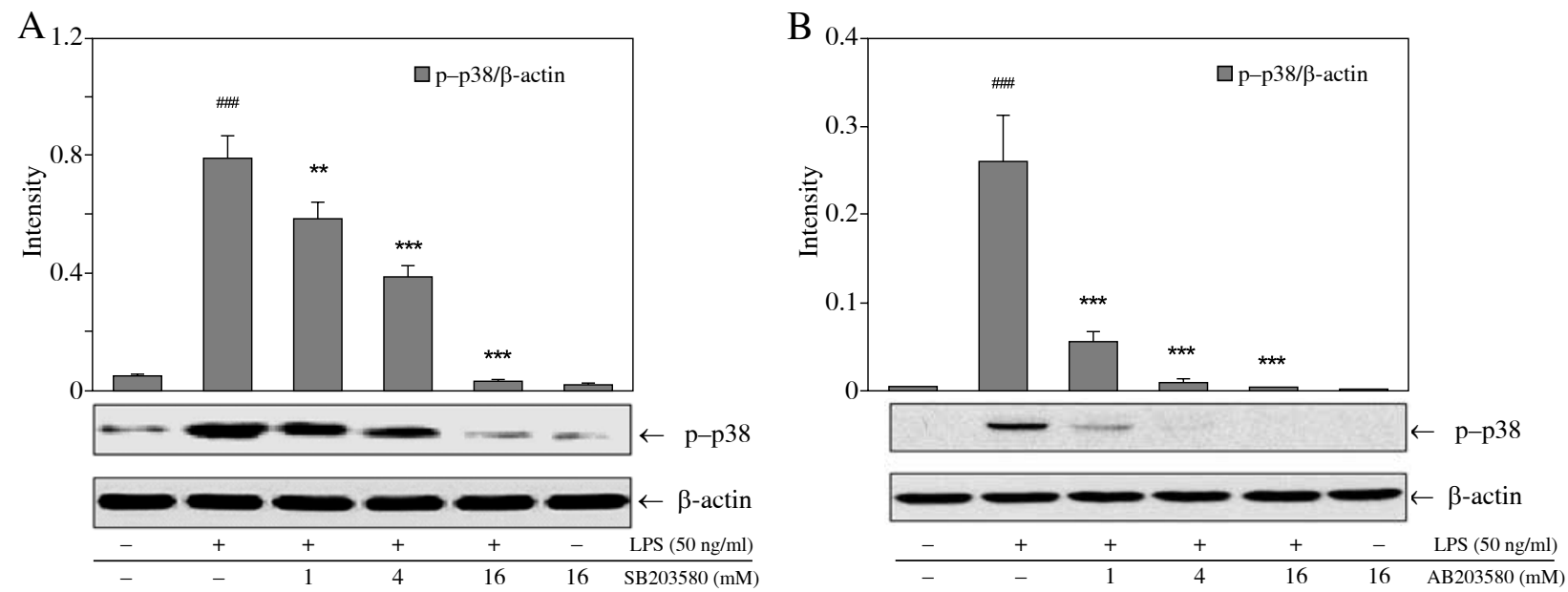

Fig. 2. The kinase inhibitory effects of SB203580 in macrophages. Cells were pretreated with SB203580 for $1 \mathrm{~h}$ and stimulated with LPS $(50 \mathrm{ng} / \mathrm{ml})$ for $30 \mathrm{~min}$. Cells were then harvested to examine the levels of p38 phosphorylation by western blotting. (A, B) Phosphorylation of p38 in RAW264.7 (A) or mouse resident peritoneal (B) macrophages;

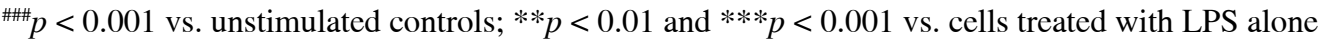
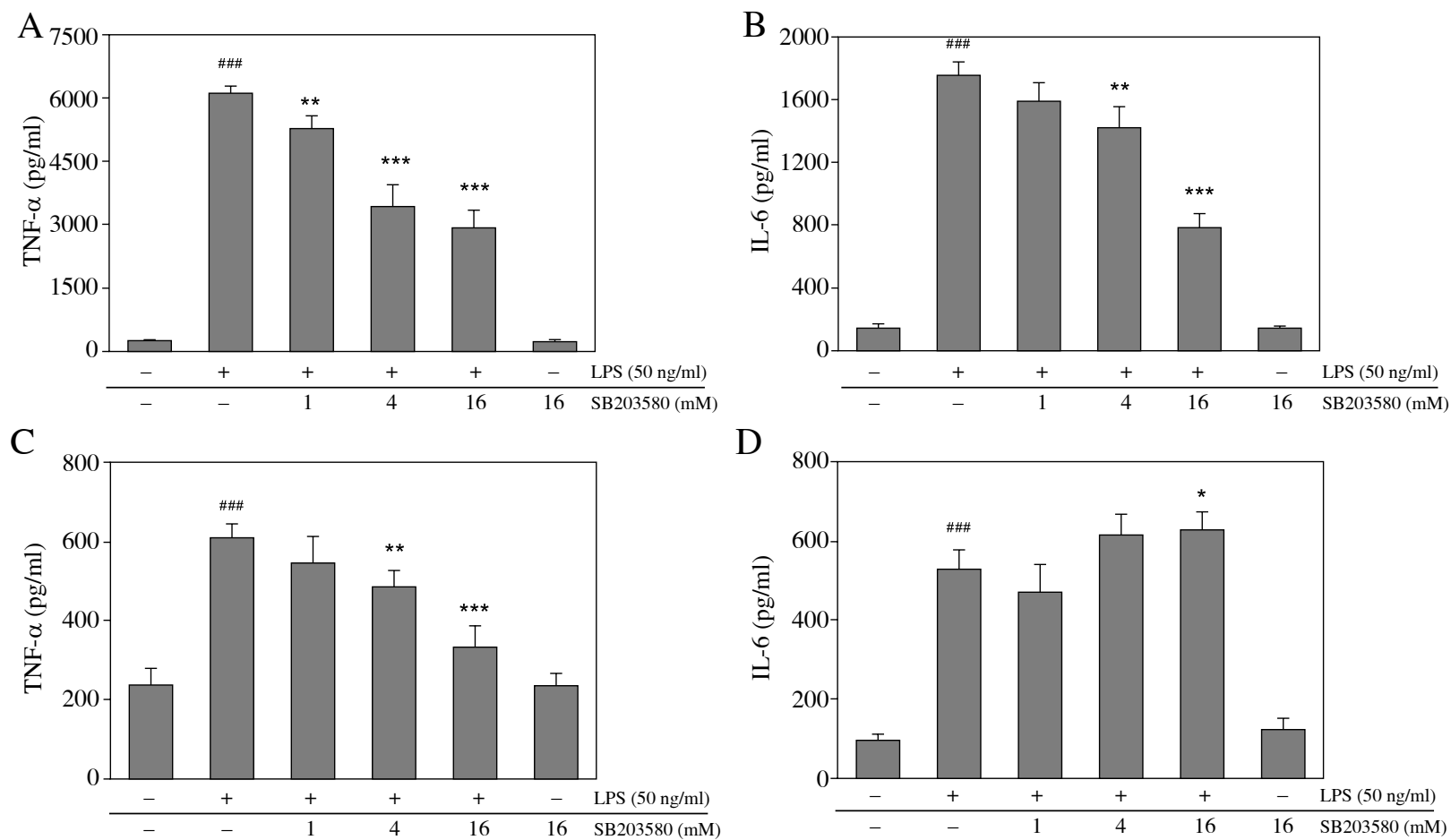

Fig. 3. SB203580 differentially modulates LPS-induced pro-inflammatory cytokine production in macrophages. Cells were pretreated with SB203580 at the indicated concentrations for $1 \mathrm{~h}$ and then were stimulated by adding LPS (50 $\mathrm{ng} / \mathrm{ml}$ ); culture supernatants were collected $12 \mathrm{~h}$ later and concentrations of TNF- $\alpha$ and IL- 6 were measured by enzyme-linked immunosorbent assay. Data shown represent (A) TNF- $\alpha$ and (B) IL-6 production by RAW264.7 macrophages, and (C) TNF- $\alpha$ and (D) IL-6 production by mouse resident peritoneal macrophages; ${ }^{\# \#} p<0.001$ vs. unstimulated controls; $* p<0.05, * * p<0.01$, and $* * * p<0.001$ vs. the LPS alone group 

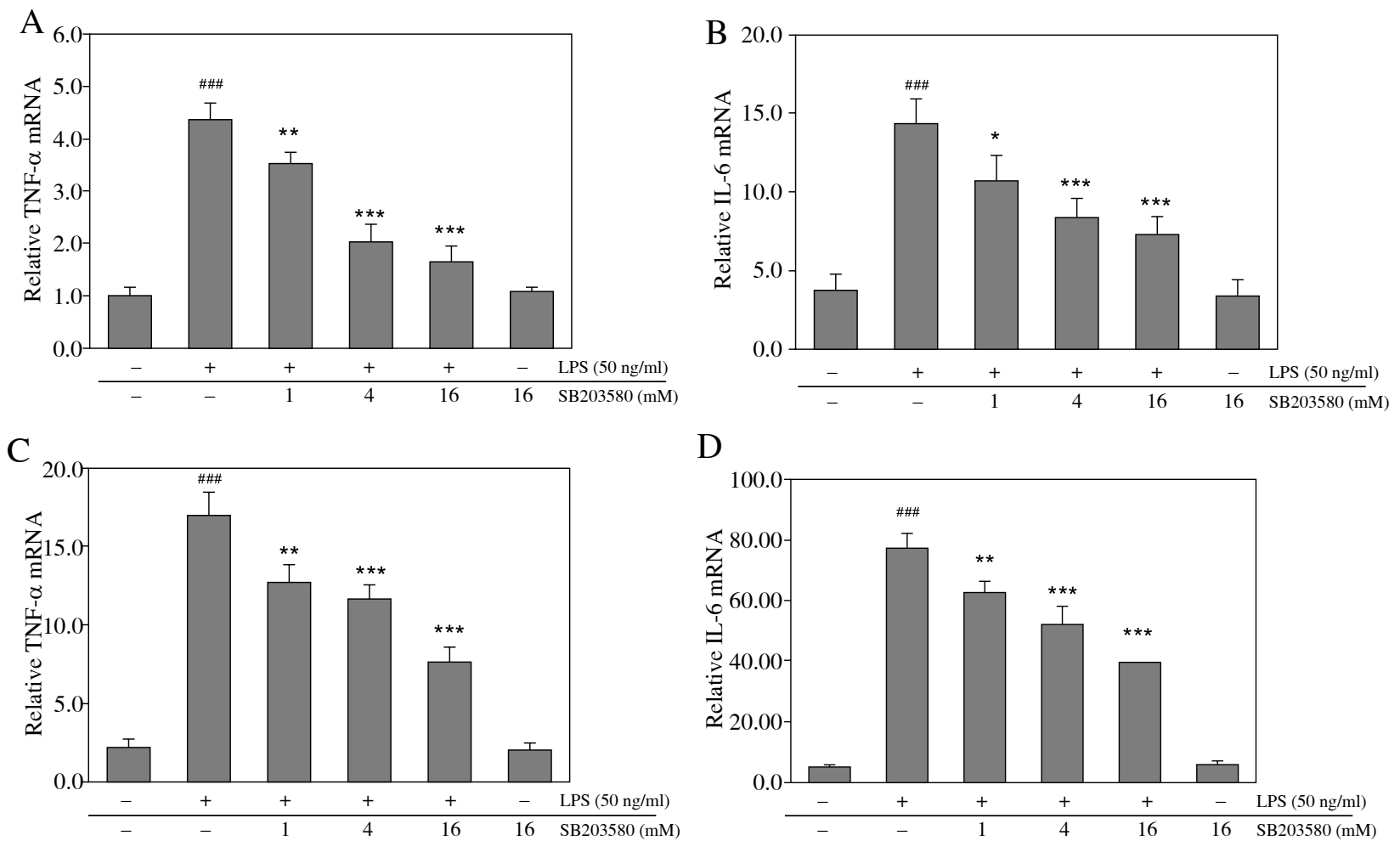

Fig. 4. SB203580 differentially regulates the transcription of LPS-induced inflammatory cytokine genes in macrophages. Cells were pretreated for $1 \mathrm{~h}$ with SB203580 and stimulated for another $4 \mathrm{~h}$ with LPS (50 ng/ml). Then, cells were collected to measure the transcript levels of TNF- $\alpha$ and IL- 6 by quantitative real-time reverse transcriptase PCR. Data shown represent (A) TNF- $\alpha$ and (B) IL-6 mRNA levels in RAW264.7 macrophages, and (C) TNF- $\alpha$ and (D) IL-6 mRNA levels in mouse resident peritoneal macrophages. For transcript quantification, the mRNA expression data were normalized to the $\beta$-actin signal using the $2^{-\Delta \Delta C T}$ method; ${ }^{\# \#} p<0.001$ vs. unstimulated controls; $* p<0.05, * * p<0.01$, and $* * * p<0.001$ vs. the LPS alone group

\section{Inhibition of p38 MAPK regulates the transcription of LPS-induced inflammatory cytokine genes in macrophages}

To investigate whether SB203580 could differentially regulate cytokine expression at the transcriptional level in RAW264.7 or mouse resident peritoneal macrophages, mRNA samples were analyzed by real-time RT-PCR to measure TNF- $\alpha$ and IL-6 transcript levels. Stimulation with LPS significantly up-regulated TNF- $\alpha$ and IL-6 mRNA transcript levels in RAW264.7 and mouse resident peritoneal macrophages, while pretreatment with SB203580 markedly abrogated this effect (Fig. 4A-D).

\section{Discussion}

Several studies have shown that MAPKs are important serine/threonine signaling kinases that can be activated by phosphorylation, resulting in cellular responses to extracellular signals that result in the modulation of gene expression. Activation of macrophages by LPS can up-regulate the phosphorylation of MAPKs, which leads to the subsequent activation of AP-1 and NF- $\kappa \mathrm{B}$; both events are involved in the expression of LPS-induced inflammatory cytokines, such as TNF- $\alpha$ and IL-6. The LPS-induced phosphorylation of p38, Erk1/2, and JNK can be blocked by specific MAPK inhibitors, such as SB203580 (a p38 inhibitor), U0126 (an Erk1/2 inhibitor), or SB600125 (a SAPK/JNK inhibitor). Previously, we showed that MAPK inhibitors could partially inhibit the LPS-induced production of inflammatory mediators, such as nitric oxide (NO), prostaglandin $\mathrm{E}_{2}\left(\mathrm{PGE}_{2}\right)$, and TNF- $\alpha$, in RAW264.7 macrophages. Herein, we studied the effects of a well-characterized p38 inhibitor, SB203580, on the ability of activated macrophages to produce inflammatory cytokines. SB203580 inhibited the p38 MAPK pathway through the down-regulation of $\mathrm{p} 38$ phosphorylation, reduced LPS-induced TNF- $\alpha$ and IL-6 mRNA transcript up-regulation and inhibited TNF- $\alpha$ and IL-6 production in RAW264.7 macrophages. By contrast, in LPS-activat- 
ed mouse resident peritoneal macrophages, SB203580 reduced TNF- $\alpha$ and IL- 6 mRNA transcription and inhibited TNF- $\alpha$ production, but failed to inhibit IL-6 production. Such differences in $\mathrm{p} 38$ dependency between different subsets of macrophages have been previously reported, but the reason for this discrepancy has not yet been established.

The regulation of gene expression in eukaryotes, at both the transcriptional and post-transcriptional levels, involve the regulation of steady-state mRNA levels and the amount of mRNA translation. The studies reported herein show that SB203580 could similarly reduce the levels of IL-6 transcription in RAW264.7 macrophages and mouse resident peritoneal macrophages. Additionally, SB203580 reduced IL-6 production in RAW264.7 macrophages, but failed to affect IL-6 production in mouse resident peritoneal macrophages. Furthermore, high concentrations of SB203580 could increase IL-6 production. Taken together, these data suggest that SB203580 differentially modulates LPS-stimulated transcription and translation of IL-6 in different types of macrophages; however, its mechanism of action remains unknown.

The MAPK inhibitor SB203580 might potentially act via several different pathways. First, steady-state mRNA levels might play a key role in the regulation of IL-6 cytokine expression. Jones et al. reported that miR-26 family microRNA can regulate target IL-6 mRNA transcripts. When the RNA-binding protein Zcchc11 adds terminal uridines to miR-26, uridylated miR-26 fails to repress IL-6 mRNA levels, which could represent a mechanism for the potentiation of IL-6 production. Although RAW264.7 and resident peritoneal macrophages were exposed to the same SB203580 compound, cell type-specific differences might influence the effects of SB203580 on steady-state levels of IL-6 mRNA transcripts. Second, SB203580 primarily acts to block the catalytic activity of p38 MAPK, but not its activation by upstream MAPK. However, if SB203580 is used at a high concentration, it can also suppress the activity of other kinases, such as protein kinase B, lymphocyte kinase, and glycogen synthase kinase 3. Suppression of protein kinase B activity might also influence post-transcriptional IL-6 expression levels.

Overall, our results show that SB203580 differentially modulates LPS-induced production of the inflammatory cytokine IL-6 in two different types of macrophages, and this regulation occurs at the IL-6 mRNA post-transcriptional stage.

This work was supported by a grant from the National Science Foundation of China (No. 81071535). This manuscript was copyedited by Elixigen (Huntington Beach, CA).

The authors declare no conflict of interest.

\section{References}

1. Nathan C (2002): Points of control in inflammation. Nature 420: 846-852.

2. Medzhitov R (2008): Origin and physiological roles of inflammation. Nature 454: 428-435.

3. Alexander C, Rietschel ET (2001): Bacterial lipopolysaccharides and innate immunity. J Endotoxin Res 7: 167-202.

4. Cuenda A, Rousseau S (2007): p38 MAP-kinases pathway regulation, function and role in human diseases. Biochim Biophys Acta 1773: 1358-1375.

5. Lee JC, Adams JL (1995): Inhibitors of serine/threonine kinases. Curr Opin Biotechnol 6: 657-661.

6. Pan X, Cao X, Li N, et al. (2014): Forsythin inhibits lipopolysaccharide-induced inflammation by suppressing JAK-STAT and p38 MAPK signalings and ROS production. Inflamm Res 63: 597-608.

7. Wang XM, Kim HP, Song R, et al. (2006): Caveolin-1 confers antiinflammatory effects in murine macrophages via the MKK3/p38 MAPK pathway. Am J Respir Cell Mol Biol 34: 434-442.

8. Shi Q, Cao J, Fang L, et al. (2014): Geniposide suppresses LPS-induced nitric oxide, PGE2 and inflammatory cytokine by downregulating NF-kappaB, MAPK and AP-1 signaling pathways in macrophages. Int Immunopharmacol 20: 298 306.

9. Kim SH, Kim J, Sharma RP (2004): Inhibition of p38 and ERK MAP kinases blocks endotoxin-induced nitric oxide production and differentially modulates cytokine expression. Pharmacol Res 49: 433-439.

10. Li X, Udagawa N, Takami M, et al. (2003): p38 Mitogen-activated protein kinase is crucially involved in osteoclast differentiation but not in cytokine production, phagocytosis, or dendritic cell differentiation of bone marrow macrophages. Endocrinology 144: 4999-5005.

11. Page TH, Brown A, Timms EM, et al. (2010): Inhibitors of p38 suppress cytokine production in rheumatoid arthritis synovial membranes: does variable inhibition of interleukin-6 production limit effectiveness in vivo? Arthritis Rheum 62: 3221-3231.

12. Ray A, Dittel BN (2010): Isolation of mouse peritoneal cavity cells. J Vis Exp 35: 1488.

13. Karin M (1992): Signal transduction from cell surface to nucleus in development and disease. FASEB J 6: 2581-2590.

14. Eyers PA, Craxton M, Morrice N, et al. (1998): Conversion of SB 203580-insensitive MAP kinase family members to drug-sensitive forms by a single amino-acid substitution. Chem Biol 5: 321-328.

15. Favata MF, Horiuchi KY, Manos EJ, et al. (1998): Identification of a novel inhibitor of mitogen-activated protein kinase kinase. J Biol Chem 273: 18623-18632.

16. DeSilva DR, Jones EA, Favata MF, et al. (1998): Inhibition of mitogen-activated protein kinase kinase blocks T cell proliferation but does not induce or prevent anergy. J Immunol 160: 4175-4181.

17. Ross J (1995): mRNA stability in mammalian cells. Microbiol Rev 59: 423-450.

18. Fabian MR, Sonenberg N, Filipowicz W (2010): Regulation of mRNA translation and stability by microRNAs. Annu Rev Biochem 79: 351-379. 
19. Jones MR, Quinton LJ, Blahna MT, et al. (2009): Zcchc11-dependent uridylation of microRNA directs cytokine expression. Nat Cell Biol 11: 1157-1163.

20. Kumar S, Jiang MS, Adams JL, et al. (1999): Pyridinylimidazole compound SB 203580 inhibits the activity but not the activation of p38 mitogen-activated protein kinase. Biochem Biophys Res Commun 263: 825-831.

21. Davies SP, Reddy H, Caivano M, et al. (2000): Specificity and mechanism of action of some commonly used protein kinase inhibitors. Biochem J 351: 95-105. 